\title{
Relación entre aprendizaje de la lectura y nivel socioeconómico en niños argentinos
}

\section{Relationship between Reading and Socioeconomic Level in frrgentine Children Relação entre aprendizagem da leitura e nível socioeconómico em crianças argentinas}

\author{
Sebastián Urquijo, Ana García Coni* \\ Universidad Nacional de Mar del Plata, Argentina \\ Débora Fernandes \\ Universidade do Vale do Sapucaí, Brasil
}

Doi: dx.doi.org/10.12804/ap133.02.2015.09

\section{Resumen}

El objetivo de este trabajo ha sido proveer datos empíricos sobre el desempeño en lectura de estudiantes de educación primaria de escuelas de gestión pública y privada de Mar del Plata, Argentina, considerando las diferencias en el nivel socioeconómico de los participantes. Se trabajó con 227 niños y niñas, de primer y tercer año de la educación primaria. Para medir el desempeño en lectura se utilizaron 10 escalas de la batería de Evaluación Neuropsicológica Infantil (ENI), cuatro de ellas sobre decodificación y seis sobre comprensión. Para evaluar el nivel socioeconómico y cultural (NSEC) de los alumnos se consideró el tipo de escuela a la que asisten y se recabó información sobre clase social, condición socioocupacional del jefe/a de familia, nivel educacional de los padres y situación ocupacional actual del jefe/a de familia de los participantes. Los resultados permitieron confirmar que el desempeño en pruebas de lectura varía en función del tipo de escuela, y se encontró en prácticamente todas las tareas administradas un desempeño inferior en estudiantes de la escuela pública, provenientes de familias de NSEC más bajo. También se estableció que la diferencia en tareas de acceso léxico se reduce con el tiempo de escolarización. En conclusión, es importante insistir en la escolarización temprana de los niños de NSEC más desfavorecidos, con el objeto de compensar los déficits que produce la escasa estimulación para la lectura y la pobreza de sus contextos alfabetizadores.

Palabras clave: lectura; aprendizaje; nivel socioeconómico y cultural; escolarización.

* Sebastián Urquijo, Centro de Investigación en Procesos Básicos, Metodología y Educación, Facultad de Psicología, Universidad Nacional de Mar del Plata (UNMdP), Argentina; Ana García Coni, Centro de Investigación en Procesos Básicos, Metodología y Educación, Facultad de Psicología, UNMdP; Débora Fernandes, Psicología, Faculdade de Ciências da Saúde, Universidade do Vale do Sapucaí (Univas), Brasil.

Esta investigación fue financiada por el Consejo Nacional de Investigaciones Científicas y Técnicas (Conicet) de Argentina. La correspondencia relacionada con este artículo debe ser dirigida a Ana García Coni, Universidad Nacional de Mar del Plata, Argentina. Correo electrónico: anagconi@gmail.com

Para citar este artículo: Urquijo, S., García Coni, A. \& Fernandes, D. (2015). Relación entre aprendizaje de la lectura y nivel socioeconómico en niños argentinos. Avances en Psicología Latinoamericana, 33(2), 303-318. doi: dx.doi.org/10.12804/ ap133.02.2015.09 


\section{fibstract}

The aim of this work was to provide empirical data about reading performance in elementary school children from public and private schools in Mar del Plata, Argentina, considering the differences in their socioeconomic status (SES). The sample consisted of $2271^{\text {st }}$ and $3^{\text {rd }}$ graders (male and female). In order to assess reading performance, we administered 10 scales from the Child Neuropsychological Assessment Set - known by the acronym ENI in Spanish-, four of them related to decoding and six related to comprehension. To assess the participants' SES, we considered the type of school (public or private) they go to, and we requested information about social class, socio-occupational status of the head of household, parents' educational level and current occupational status of the head of household. Our results confirm that reading performance varies with type of school and SES, as students that go to a public school and come from lower socioeconomic backgrounds underperformed in almost all the tests. We also found that differences in lexical access tasks are reduced during schooling. To conclude, we insist in the early schooling of children that come from disadvantaged socioeconomic backgrounds, in order to mitigate the deficits caused by a weak reading stimulation and a poor literacy environment.

Key words: reading; learning; socioeconomic status; schooling.

\section{Resumo}

O objetivo deste trabalho tem sido o de prover dados empíricos sobre o desempenho em leitura de estudantes de educação primária de escolas de gestão pública e privada de Mar del Plata, Argentina, considerando as diferenças no nível socioeconómico dos participantes. Trabalhou-se com 227 crianças, de 1ro e 3ro ano da educação primária. Para mensurar o desempenho em leitura utilizaram-se 10 escalas da bateria de Avaliação Neuropsicológica Infantil (ANI), quatro delas sobre decodificação e seis acerca de compreensão. Para avaliar o nível socioeconômico e cultural (NSEC) dos alunos considerou-se o tipo de escola ao que assistem, e se obteve informação sobre classe social, condição sócio-ocupacional do chefe de família, nível educacional dos pais e situação ocupacional atual do chefe de família dos participantes. Os resultados permitiram confirmar que o desempenho em provas de leitura varia em função do tipo de escola, encontrando em praticamente todas as tarefas administradas um desempenho inferior em estudantes da escola pública, proveniente de famílias de NSEC mais baixo. Também estabeleceu-se que a diferença em tarefas de acesso léxico se reduz com o tempo de escolarização. Conclui-se na importância de insistir na escolarização prematura das crianças de NSEC mais desfavorecido, com o objeto de compensar os déficits que produz a escassa estimulação para a leitura e a pobreza de seus contextos alfabetizadores.

Palavras-chave: leitura; aprendizagem; nível socioeconómico e cultural; escolarização.

El aprendizaje de la lectoescritura constituye una de las tareas más importantes de la educación primaria, dado que la habilidad de leer y de escribir incide directamente en el éxito y en el fracaso de niños y adolescentes en la escuela y fuera de ella (Urquijo, 2009). En este sentido, la obtención de información acerca de los contenidos de distintas asignaturas y áreas de conocimiento está estrechamente vinculada con el desarrollo de las competencias lectoras (De los Reyes Aragón, Lewis Harb \& Peña Ortiz, 2008). Al respecto, Hallahan, Kauffman y Lloyd (1999) señalan que los déficits académicos en la lectura son los más comunes en los estudiantes con dificultades de aprendizaje, y, por lo tanto, evaluar y compensar las dificultades en dicha área es una tarea ineludible de las instituciones educativas.

En un sentido más amplio, escuchar, hablar, leer y escribir constituyen un continuo de habilidades psicolingüísticas que garantizan la competencia comunicativa (Snow, 1983; Urquijo, 2009). Desde la perspectiva de la psicología cognitiva, se ha establecido que los procesos implicados en la lectura, denominados procesos lectores, son básicamente 
la identificación de letras, el reconocimiento de palabras y la comprensión lectora (Urquijo, 2009). De acuerdo con Defior Citoler (1996), la identificación de letras y palabras está implicada en la decodificación lectora, que es un proceso de menor nivel que el de la comprensión.

Decodificar implica discriminar letras - y grupos de letras - , reconocer palabras escritas, acceder a su significado y atribuirles una pronunciación. Requiere habilidades tanto visuales como lingüísticas (fonológicas, semánticas y pragmáticas) y es considerado un proceso de bajo nivel, que se automatiza con el ejercicio, a medida que el lector va haciéndose más avezado en la tarea. Los trastornos en el desarrollo de estas habilidades reciben el nombre de trastornos específicos del aprendizaje de la lectura.

De acuerdo con Defior Citoler (1996), el reconocimiento de palabras debe convertirse en un proceso automático, para poder destinar más recursos atencionales a los procesos de nivel superior, dedicados a la comprensión. La autora afirma que para aprender a leer, los niños deben dominar los tipos de señales que proporciona el texto. El primer tipo de señal está conformado por los símbolos gráficos que representan los sonidos; depende de que los niños conozcan las reglas de conversión grafema-fonema (RCGF)y establezcan las correspondencias necesarias para interpretar los símbolos de la escritura. Este proceso implica tanto una actividad perceptivo-visual - discriminar e identificar las letras - como fonológica - conocer las correspondencias sonoras de las letras-.

También hay señales contextuales, dado que en un texto las palabras están acompañadas de otras palabras que pueden oficiar de señales que contribuyan a su decodificación, y el tercer tipo de señales es el estructural, que consiste "en una serie de convenciones características del lenguaje escrito, como son que se escribe y se lee de izquierda a derecha $[. .$.$] que los textos están estructurados en$ forma narrativa, expositiva, etc." (Defior Citoler, 1996, p. 66).
De este modo, el dominio de las señales del texto se vincula estrechamente con las habilidades fonológicas del lector y con la familiaridad con la lectura que pueda aportar su ambiente. Respecto a la conciencia fonológica, esta se incluye dentro de la conciencia lingüística, y constituye una habilidad metalingüística que implica la reflexión y el control deliberado sobre el lenguaje (Garton \& Pratt, 1991; Moraso \& Duro, 2004). Específicamente, la conciencia fonológica consiste en la reflexión consciente sobre los sonidos de la propia lengua (Defior Citoler, 1994); en otras palabras, supone la toma de conciencia de que en el lenguaje oral hay una serie de secuencias fonológicas que pueden descomponerse en secuencias más simples, e incluye la capacidad para manipular esas secuencias (De los Reyes et al., 2008).

Con respecto a la importancia de estar familiarizado con la lectura, se considera que el conocimiento que poseen los niños acerca del material gráfico incluye tanto el conocimiento del objeto libro como el conocimiento de la escritura como sistema, que abarca la comprensión de su funcionalidad - es decir, saber que un texto posee y transmite un significado para un otro distante o ausente, conocer la direccionalidad de la escritura y conocer el aspecto visual de un texto- (Olinghouse \& Graham, 2009), y el conocimiento de las letras, que implica conocer el grafismo, el nombre o el fonema que representan algunas letras (Guardia, 2003; Vellutino \& Scanlon, 2002).

Al retomar la caracterización de los procesos particulares de la lectoescritura, cabe señalar que cuando hay problemas en la decodificación, se dificulta la comprensión del texto, que es un proceso de más alto nivel. No puede lograrse una lectura competente, que incluye la comprensión, si no se decodifica fluidamente. A su vez, la decodificación fluida no solo implica dominio de las RCGF, sino también su uso automatizado. No obstante, la automatización de los procesos de acceso léxico no basta para asegurar un proceso eficiente de comprensión. 
Con respecto a la comprensión de textos, de acuerdo con el modelo de Kintsch y Van Dijk (citado en García Madruga, 2006), no es un proceso a todo o nada, sino que consta de tres niveles: (a) la representación lingüística superficial, que reproduce de manera exacta las características gramaticales del texto, con consecuencias irrelevantes desde el punto de vista psicológico, ya que lo que perdura en la mente del lector es el significado y no la superficie del texto; (b) la base del texto o representación proposicional, que da cuenta de las ideas a las que el texto hace referencia, considerando el modo en que cada proposición se relaciona con las que ya han sido presentadas, y (c) el modelo mental de la situación, en el que los conocimientos del sujeto se integran a la información semántica del texto y se obtiene su organización lógica.

García Madruga (2006) sostiene que en el nivel proposicional el lector se representa el significado del texto a través de la microestructura, que consiste en el conjunto de ideas o proposiciones que se relacionan entre sí por la repetición de argumentos que tienen una referencia común. Sin embargo, la coherencia que resulta de compartir una referencia no alcanza para acceder al significado acabado del texto. El significado global está por encima de la necesidad del texto de referirse a unas mismas personas, objetos o situaciones. De modo que la microestructura puede usarse como base sobre la cual construir la macroestructura o representación del significado global del texto. En otras palabras, a partir de las proposiciones (microestructura) se forman las macroproposiciones de niveles cada vez más altos, que representan el tema o la idea general, y son inferidas mediante la utilización de macrorreglas. Estas son aplicadas a partir del conocimiento del lector y del contenido del texto, con el objetivo de reducir y organizar la información de la microestructura suprimiendo el número de proposiciones y manteniendo las más relevantes, e incorporando nuevas proposiciones por medio de la generalización o construcción. Según León y García Madruga (1991), la aplicación de estas reglas es una tarea controlada, aprendida, y que depende de los propósitos y conocimientos del lector.

En suma, el lector no solo lee e interpreta palabras y frases, sino que construye un modelo coherente e integrado del texto global, que supone la utilización activa de esquemas temáticos y formales que guían la comprensión. El individuo que realiza una lectura comprensiva opera simultáneamente en todos los niveles de procesamiento.

Se suele categorizar como microprocesos aquellos directamente relacionados con la decodificación más o menos mecánica del texto (reconocimiento de letras, construcción silábica y codificación de palabras), y se suelen considerar macroprocesos las operaciones de alto nivel asociadas con la comprensión del texto (integración de proposiciones en esquemas, inferencias y uso de metas en la lectura). Los dos niveles son esenciales para que un individuo pueda asumirse como un buen lector, lo cual supone una considerable carga cognitiva, dado que se deben ejecutar simultáneamente las operaciones de decodificación y de comprensión. Las diferencias individuales que se hallan entre buenos y malos lectores se deben al grado de eficiencia de ambos procesos (Urquijo, 2009).

En este sentido, de acuerdo con Sternberg (1996), aprender a leer requiere dominar dos formas básicas de procesos: los léxicos y los de comprensión. Los primeros se utilizan para identificar letras y palabras y para activar información relevante sobre esas palabras en la memoria. Los segundos, a su vez, se usan para entender el texto como una totalidad, estableciendo relaciones entre los significados de las palabras. Así, la comprensión supone un rol activo por parte del lector, quien construye el significado de los textos de acuerdo con su bagaje cultural, sus metas y la situación de lectura (Gunning, 1998).

De lo expuesto se deduce que la lectura exige una serie de requisitos cognitivos, como la atención, la memoria, el lenguaje y la abstracción. La atención, por ejemplo, es indispensable para lograr 
una adecuada decodificación de los estímulos y una correcta comprensión del texto (Rosselli, Matute \& Ardila, 2006). Además, se asocian con el aprendizaje de la lectura numerosos factores ambientales, por ejemplo, la exposición a la lectura, el contexto alfabetizador, las características socioeconómicas y culturales de los hogares de los aprendices y el desarrollo de una actitud y una motivación positivas (Bracken \& Fischel, 2008; Molfese, Modglin \& Molfese, 2003; Urquijo, 2009). Así, la disponibilidad de portadores de texto en los hogares, los hábitos de lectura de los padres, la lectura temprana de cuentos a los niños, etc., son variables que se vinculan con la alfabetización temprana (Andrés, Canet-Juric, Richard's, Introzzi \& Urquijo, 2010; Andrés, Navarro Guzmán, Urquijo \& Cedeño García, 2010; Graves, Juel \& Graves, 2000; Hourigan, 1994; Marder, Querejeta, Piacente, Resches \& Urrutia, 2005; Piacente, Granato, Talou \& Marder, 2000; Snow \& Beals, 2006).

En este sentido, aquellas familias que hacen un uso cotidiano de la lectura y la escritura -incluida tanto la comprensión como la producción de textos - brindan a sus hijos conocimientos sobre lo impreso y sobre la funcionalidad y el uso del lenguaje escrito (Purcell-Gates, 1996). Al respecto, se considera que el capital cultural familiar incide significativamente en el logro escolar del alumno y se toma, comúnmente, como medida de ese capital la cantidad de libros existente en el hogar y el uso que se les da. Así, de acuerdo con el Ministerio de Educación de la República Argentina (DiNIECE, 2012), entre los alumnos de sexto año de la escuela primaria que menos libros declaran tener en su casa, solamente el $12.3 \%$ obtiene un desempeño alto en lengua; mientras que el $36.5 \%$ de ellos no consigue superar el nivel más bajo de desempeño. En contraste, solo el $19.77 \%$ de los estudiantes que declara tener una gran cantidad de libros en su hogar presenta un nivel bajo de desempeño en lengua, en tanto el $32.11 \%$ obtiene desempeños altos.

En síntesis, el lector aplica sus estrategias y conocimientos —no solo generales, sino también relacionados con la manera en que se organizan los textos - en la tarea de reconocer y seleccionar las ideas más importantes, y de tomar las indicaciones y los distintos tipos de información que se incluyen en los textos, como los títulos, los resúmenes, las numeraciones, etc. De este modo, las demandas cognitivas de la alfabetización son complejas; a la dificultad de la decodificación alfabética se le une la necesidad de contar con ciertas destrezas conceptuales, motivaciones y hábitos. En otras palabras, el proceso de leer y escribir no se agota en el desarrollo de habilidades básicas; también constituye una práctica social y comunicativa que está en la base de las habilidades discursivas necesarias para alcanzar múltiples propósitos (Gee, 1999; Hourigan, 1994; Lee, 1995; Moje, Dillon \& O’Brien, 2000; Scribner \& Cole, 1981; Street, 1984).

Por ende, la lectoescritura es un sistema abstracto que demanda un arduo trabajo intelectual por parte del sujeto que intenta acceder a ella. Según Baquero (1996), parecería que el desarrollo exige un grado cada vez mayor de conciencia y voluntad para dominar los objetos de conocimiento de la cultura, y un uso de instrumentos de mediación cada vez más descontextualizados. En este sentido, para Vigotsky (1988) las funciones psicológicas más avanzadas implican una reestructuración del comportamiento del sujeto, que le permite un mayor control sobre sus operaciones intelectuales.

Debe tenerse en cuenta que los niños que viven en ambientes socioculturales pobres tienden a tener un desempeño lector más limitado y mayores dificultades en el aprendizaje de la lectura que los niños que viven en ambientes más ricos (Molfese et al., 2003; Urquijo, 2009; Wigfield \& Asher, 2002). En este sentido, el nivel socioeconómico de los cuidadores de los niños es estimado como un predictor fuerte del desempeño académico en tareas de lectura y escritura al inicio del primer grado (Magnuson, 2007; National Assessment of Educational Progress, 1991). Asimismo, la disponibilidad de recursos materiales vinculados con la lectura tiene relación con el nivel cultural, laboral 
y de ingresos de la familia (Bradley \& Corwyn, 2002; Fernández \& Salvador, 1994; Ruiz de Miguel, 1999). Al respecto, de acuerdo con datos del Operativo Nacional de Evaluación 2010, realizado en Argentina (DiNIECE, 2012), del total de alumnos de sexto año de educación primaria de muy bajo nivel económico, apenas el 10.6\% alcanza un desempeño alto en la asignatura Lengua, mientras que ese porcentaje asciende al $34.1 \%$ entre los alumnos de muy alto nivel económico.

En suma, tanto el nivel laboral como el nivel educativo y de ingresos de los padres se han asociado con el funcionamiento cognitivo de niños escolares y preescolares (Farah et al., 2006; Fueyo, 1990; Gordon \& Greenidge, 1999; Hackman \& Farah, 2009; Lipina, Martelli, Vuelta, Injoque-Ricle \& Colombo, 2004; Magnuson, 2007; Recart-Herrera, Mathiesen \& Herrera-Garbarini, 2005). Así, las investigaciones en el campo educativo han demostrado reiteradamente que el aprendizaje del alumno se asocia de manera estrecha con su origen social, de modo que a mayores recursos económicos de la familia, mayores probabilidades de que el estudiante consiga desempeños altos (DiNIECE, 2012).

Matute, Sanz, Gumá, Rosselli y Ardila (2009) señalan que el estatus socioeconómico se asocia con algunas características de las escuelas a las que asisten los niños. Así, se ha encontrado que la calidad de la educación recibida en las escuelas depende de variables como el gasto destinado a cada alumno, la calidad de los maestros, la proporción maestro-alumnos, la presencia de facilidades especiales (laboratorios de ciencia, equipos de cómputo, etc.), la cantidad de días asistidos a la escuela en el año y las características de los estudiantes (historia educacional, aspiraciones, etc.) (véase Manly, Schupf, Tang, Weiss \& Stern, 2007).

Datos provenientes del Ministerio de Educación de la República Argentina (DiNIECE, 2009) sobre los niveles de desempeño en Lengua de alumnos de tercer año de educación primaria confirman la existencia de diferencias marcadas entre los alumnos del sistema urbano privado $(63.7 \%$ alcanzan un nivel alto; $23.5 \%$, un nivel medio, y $12.8 \%$, un nivel bajo) y los alumnos del sistema urbano estatal (32.4\% alcanzan un nivel alto; 33.4\%, nivel medio, y $34.1 \%$, nivel bajo). Estos datos muestran que existe una brecha en el rendimiento de los usuarios de ambos sistemas, lo cual resulta preocupante si se tiene en cuenta, sobre todo, que la función de la escuela pública debería ser la de propiciar la equidad y la igualdad de oportunidades en la población, proveyendo mayor formación a quienes no la han obtenido en su medio.

Es probable que las diferencias de los desempeños en Lengua se deban, al menos en parte, a diferencias en el aprendizaje de la lectoescritura; sin embargo, no abundan datos precisos sobre las diferencias específicas que los alumnos de los sistemas público y privado presentan en dicho aprendizaje. Por lo tanto, este trabajo pretende proveer datos empíricos sobre el desempeño en lectura de un grupo de estudiantes de primer y tercer año del ciclo básico de la educación primaria de escuelas de gestión pública y privada del área metropolitana de la ciudad de Mar del Plata, Argentina, considerando particularmente las diferencias en el nivel socioeconómico de los participantes.

\section{Materiales y métodos}

\section{Participantes}

Se trabajó con una muestra definitiva de 227 niños y niñas (117 de sexo femenino y 110 de sexo masculino), alumnos de primer (promedio de edad $=82$ meses, $D S=3$ meses) y tercer año (promedio de edad $=107$ meses, $D S=4$ meses) del ciclo básico de la educación primaria de escuelas públicas y privadas de la ciudad de Mar del Plata, Argentina. Se obtuvieron datos de 111 alumnos de una escuela pública escogida al azar entre las 17 escuelas de la Secretaría de Educación de la Municipalidad de General Pueyrredón - 61 alumnos de primero (28 niñas y 33 niños) y 50 alumnos de tercero (25 niñas y 25 niños) - y de 116 alumnos de dos 
escuelas privadas seleccionadas de manera intencional, una de ellas bilingüe, con doble escolaridad, y otra con escolaridad simple —66 alumnos de primero (40 niñas y 26 niños) y 50 alumnos de tercero (24 niñas y 26 niños $)$ - . En cada escuela se seleccionaron al azar los cursos y se evaluó a todos los alumnos cuyos padres hubieran prestado el consentimiento escrito para participar en la investigación. Asimismo, los niños dieron su asentimiento de forma oral y fueron entrevistados en horario escolar, en salas de las escuelas destinadas para tal fin. Durante el periodo de evaluación, algunos de los niños no asistieron a la escuela y otros no completaron correctamente las pruebas. Se excluyeron de la muestra niños y niñas repetidores y con problemas o trastornos específicos del aprendizaje.

\section{Instrumentos}

Para evaluar el desempeño en lectura se utilizaron 10 escalas de la batería de Evaluación Neuropsicológica Infantil (ENI) (Matute, Rosselli, Ardila \& Ostrovsky-Solís, 2007):

Lectura de sílabas. El niño debe leer ocho sílabas.

Lectura de palabras. El niño debe leer ocho palabras.

Lectura de no palabras. El niño debe leer ocho palabras sin sentido.

Lectura de oraciones. Precisión. El niño debe leer en voz alta 10 oraciones que incluyen instrucciones (e. g., "Señala un avión grande").

Lectura de oraciones. Comprensión. Se evalúa si el niño realiza la instrucción de manera correcta inmediatamente después de haberla leído.

Precisión en la lectura de un texto en voz alta. Se le presenta al niño un cuento y se le pide que lo lea en voz alta. Se registran las palabras leídas con error.

Tiempo de lectura de un texto en voz alta. Se le pide al niño que lea en voz alta un texto y se calcula la velocidad de lectura.
Comprensión de la lectura de un texto en voz alta. Una vez que el niño ha leído los cuentos de la prueba anterior se le hacen preguntas de comprensión.

Tiempo de lectura silenciosa de un texto. Se le pide al niño que lea en silencio un texto y se calcula la velocidad de lectura.

Comprensión de la lectura silenciosa de un texto. Una vez que el niño ha leído el texto de la prueba anterior se le hacen preguntas de comprensión.

Para evaluar el nivel socioeconómico y cultural (NSEC) de los alumnos se consideró el tipo de escuela al que asisten, debido a las marcadas diferencias existentes entre los alumnos que concurren a escuelas privadas y los que concurren a escuelas públicas en Argentina (DiNIECE, 2009). Además, se utilizaron los criterios propuestos por Torrado (1992) y se recabó información sobre la clase social, la condición sociocupacional del jefe/a de familia, el nivel educacional de los padres y la situación ocupacional actual del jefe/a de familia de los participantes.

\section{Procedimiento}

Se solicitó el consentimiento informado de los padres o cuidadores de los niños, en el cual se explicaba detalladamente en qué consistía la evaluación. Todas las pruebas fueron administradas por profesionales y alumnos avanzados de la carrera de Psicología, especialmente entrenados para la tarea, en aulas de las instituciones educativas, de forma individual y durante aproximadamente 30 minutos.

\section{Resultados}

Con respecto al NSEC de los estudiantes, se hallaron diferencias claras en todas las dimensiones: en cuanto a la clase social, el $70 \%$ de las familias que envía a sus hijos a las escuelas de gestión privada pertenece a la clase media y poco más del $25 \%$ de los sujetos que asisten a la escuela pública selec- 
cionada pertenece a esa categoría; más de un $70 \%$ de los alumnos de esa escuela pertenece a familias de clase obrera, en tanto en las escuelas privadas ese segmento solo representa un $21 \%$. Acerca del nivel educativo, menos de un $10 \%$ de los padres de los alumnos de la escuela pública ha asistido a la universidad y casi un $60 \%$ no ha asistido al secundario; en las escuelas privadas, en cambio, el $66 \%$ de los padres de los alumnos ha asistido a la universidad y más del $50 \%$ ha obtenido un título. Se halla el mismo patrón en el caso de las madres de los estudiantes (tablas 1, 2 y 3 ).

Tabla 1

Clase social y condición socioocupacional del jefela de familia según tipo de escuela

\begin{tabular}{lcc}
\hline \multicolumn{1}{c}{ Clase social de la familia } & Escuela pública (\%) & Escuelas privadas $(\%)$ \\
\hline Clase alta & 26.13 & 8.62 \\
Clase media & 73.87 & 69.83 \\
Clase obrera & & 21.55 \\
\hline Condición sociocupacional & - & 8.62 \\
\hline Directores de empresa & 1.80 & 33.62 \\
Profesional en función específica & 1.80 & 10.34 \\
Propietario de pequeña empresa & 7.21 & 9.48 \\
Cuadros técnicos & 7.21 & 6.90 \\
Pequeños productores autónomos & 7.21 & 6.90 \\
Empleados administrativos y vendedores & 7.21 & 9.48 \\
Trabajadores especializados autónomos & 22.52 & 6.03 \\
Obreros calificados & 22.52 & 4.31 \\
Obreros no calificados & 9.01 & 1.72 \\
Peones autónomos & 8.11 & - \\
Empleados domésticos & 5.41 & 2.59 \\
Sin especificar & & \\
\hline
\end{tabular}

Tabla 2

Nivel educativo de los padres según tipo de escuela

\begin{tabular}{lcccc}
\hline \multirow{2}{*}{$\begin{array}{c}\text { Nivel educativo del } \\
\text { progenitor }\end{array}$} & \multicolumn{2}{c}{ Padre } & \multicolumn{2}{c}{ Madre } \\
\cline { 2 - 5 } & Escuela pública (\%) & Escuelas privadas (\%) & Escuela pública (\%) & Escuelas privadas (\%) \\
\hline Analfabeto & 5.41 & .86 & 3.60 & - \\
Lee y escribe & 4.50 & - & 4.50 & - \\
Primario incompleto & 17.12 & 1.72 & 14.41 & .86 \\
Primario completo & 32.43 & 6.90 & 34.23 & 3.45 \\
Secundario incompleto & 9.01 & 5.17 & 11.71 & 6.90 \\
Secundario completo & 22.52 & 19.83 & 21.62 & 20.69 \\
Universitario incompleto & 2.70 & 14.66 & 2.70 & 16.38 \\
Universitario completo & 6.31 & 50.86 & 7.21 & 51.72 \\
\hline
\end{tabular}


Tabla 3

Situación ocupacional actual del jefela de familia según tipo de escuela

\begin{tabular}{lcc}
\hline \multicolumn{1}{c}{ Situación ocupacional } & $\begin{array}{c}\text { Escuela } \\
\text { pública (\%) }\end{array}$ & $\begin{array}{c}\text { Escuelas pri- } \\
\text { vadas (\%) }\end{array}$ \\
\hline No trabaja & 4.50 & 2.59 \\
Dueño & 16.22 & 30.17 \\
Empleado & 64.86 & 37.07 \\
Trabaja por cuenta propia & 9.01 & 30.17 \\
Trabaja sin sueldo fijo & .90 & - \\
Trabaja en servicio doméstico & 4.50 & - \\
\hline
\end{tabular}

Con el objeto de determinar si las diferencias de los indicadores de NSEC resultaban estadísti- camente significativas, se sometieron los datos a una prueba no paramétrica de comparación para dos muestras independientes (U de Mann-Whitney), en función del tipo de escuela. Los resultados indican que las diferencias resultaron estadísticamente significativas con un $p<.001$ para el nivel educativo del padre y de la madre, la clase social y la condición sociocupacional.

A continuación se presentan los estadísticos descriptivos de los resultados obtenidos en las pruebas de lectura, discriminados por año escolar y tipo de escuela (tabla 4).

En primer lugar, se debe destacar que el número de sujetos de primer año de la escuela pública que consiguió completar las pruebas de lectura de

Tabla 4

Estadísticos descriptivos de las variables en estudio, discriminadas por año escolar y tipo de escuela

\begin{tabular}{|c|c|c|c|c|c|c|c|}
\hline \multirow{2}{*}{ Año } & \multirow{2}{*}{ Pruebas de lectura } & \multicolumn{3}{|c|}{ Escuela pública } & \multicolumn{3}{|c|}{ Escuelas privadas } \\
\hline & & $N$ & Media & $D E$ & $N$ & Media & $D E$ \\
\hline \multirow{10}{*}{$1^{\mathrm{o}}$} & Lectura de sílabas & 60 & .90 & 2.12 & 66 & 6.35 & 2.36 \\
\hline & Lectura de palabras & 60 & 1.73 & 3.20 & 66 & 8.83 & 2.17 \\
\hline & Lectura de no palabras & 60 & .85 & 2.19 & 66 & 6.24 & 2.26 \\
\hline & $\begin{array}{l}\text { Lectura de oraciones. } \\
\text { Precisión }\end{array}$ & 60 & 1.20 & 3.10 & 66 & 7.58 & 3.12 \\
\hline & $\begin{array}{l}\text { Lectura de oraciones. } \\
\text { Comprensión }\end{array}$ & 60 & .65 & 1.81 & 64 & 5.67 & 2.73 \\
\hline & $\begin{array}{l}\text { Lectura en voz alta. } \\
\text { Tiempo }\end{array}$ & 9 & 169.56 & 109.50 & 64 & 120.20 & 118.30 \\
\hline & $\begin{array}{l}\text { Lectura en voz alta. } \\
\text { Errores }\end{array}$ & 9 & 2.78 & 2.72 & 64 & 3.58 & 6.36 \\
\hline & $\begin{array}{l}\text { Lectura en voz alta. } \\
\text { Comprensión }\end{array}$ & 8 & 3.25 & 2.43 & 64 & 4.22 & 2.80 \\
\hline & $\begin{array}{l}\text { Lectura silenciosa. } \\
\text { Tiempo }\end{array}$ & 8 & 395.00 & 388.17 & 60 & 230.63 & 157.49 \\
\hline & $\begin{array}{l}\text { Lectura silenciosa. } \\
\text { Comprensión }\end{array}$ & 7 & 2.57 & 1.71 & 60 & 1.63 & 1.67 \\
\hline \multirow{3}{*}{$3^{\circ}$} & Lectura de sílabas & 50 & 7.38 & .94 & 50 & 7.76 & 0.47 \\
\hline & Lectura de palabras & 50 & 9.82 & .56 & 50 & 10.06 & 0.62 \\
\hline & Lectura de no palabras & 50 & 7.38 & .92 & 50 & 7.48 & 0.81 \\
\hline
\end{tabular}




\begin{tabular}{|c|c|c|c|c|c|c|c|}
\hline \multirow{2}{*}{ Año } & \multirow{2}{*}{ Pruebas de lectura } & \multicolumn{3}{|c|}{ Escuela pública } & \multicolumn{3}{|c|}{ Escuelas privadas } \\
\hline & & $N$ & Media & $D E$ & $N$ & Media & $D E$ \\
\hline \multirow{7}{*}{$3^{\circ}$} & $\begin{array}{l}\text { Lectura de oraciones. } \\
\text { Precisión }\end{array}$ & 50 & 9.60 & .70 & 50 & 9.68 & 0.84 \\
\hline & $\begin{array}{l}\text { Lectura de oraciones. } \\
\text { Comprensión }\end{array}$ & 50 & 6.34 & 1.63 & 50 & 8.10 & 1.50 \\
\hline & $\begin{array}{l}\text { Lectura en voz alta. } \\
\text { Tiempo }\end{array}$ & 50 & 128.78 & 45.38 & 50 & 74.94 & 24.79 \\
\hline & $\begin{array}{l}\text { Lectura en voz alta. } \\
\text { Errores }\end{array}$ & 50 & 4.70 & 4.53 & 50 & 1.28 & 1.49 \\
\hline & $\begin{array}{l}\text { Lectura en voz alta. } \\
\text { Comprensión }\end{array}$ & 50 & 3.02 & 1.68 & 50 & 5.04 & 1.34 \\
\hline & $\begin{array}{l}\text { Lectura silenciosa. } \\
\text { Tiempo }\end{array}$ & 50 & 121.10 & 54.07 & 49 & 73.57 & 26.10 \\
\hline & $\begin{array}{l}\text { Lectura silenciosa. } \\
\text { Comprensión }\end{array}$ & 50 & 2.70 & 1.83 & 49 & 3.37 & 1.99 \\
\hline
\end{tabular}

un texto en voz alta y en silencio fue mucho más bajo que el de los sujetos de la escuela privada: tan solo nueve niños de la escuela pública realizaron la lectura en voz alta (y solo ocho realizaron la prueba de comprensión), así como solamente ocho participantes hicieron la lectura silenciosa (y solo siete completaron la prueba de comprensión).

Si bien se puede observar un mejor desempeño de los niños de tercer año en relación con los de primero, y de los de la escuela privada respecto de los de la pública, se realizaron pruebas $t$ de comparación de medias para dos muestras independientes, considerando el tipo de escuela, con el objeto de determinar si las diferencias entre los alumnos de la escuela pública y de las escuelas privadas resultaban estadísticamente significativas. En la tabla 5 se presentan los resultados y confirman que las diferencias observadas entre los alumnos de la escuela pública y los de las escuelas privadas son estadísticamente significativas, con algunas salvedades. Los alumnos de primer año de las escuelas privadas obtuvieron rendimientos superiores a los de los alumnos de primer año de la escuela pública en las tareas de lectura de sílabas, palabras y no palabras, y en las tareas de precisión y comprensión
Tabla 5

Prueba $\mathrm{t}$ de muestras independientes para comparar el rendimiento de alumnos de $1^{\text {er }}$ y $3^{\text {er }}$ año de escolaridad pública y privada en pruebas de lectura

\begin{tabular}{|c|c|c|c|c|}
\hline 是 & Pruebas de lectura & $t$ & $\begin{array}{l}\text { Diferencia } \\
\text { de medias }\end{array}$ & $\begin{array}{c}\text { Error } \\
\text { típico de la } \\
\text { diferencia }\end{array}$ \\
\hline & Lectura de sílabas & $-13.57^{* *}$ & -5.45 & 0.40 \\
\hline & Lectura de palabras & $-14.65^{* *}$ & -7.10 & 0.48 \\
\hline & Lectura de no palabras & $-13.57^{* *}$ & -5.39 & 0.40 \\
\hline & $\begin{array}{l}\text { Lectura de oraciones. } \\
\text { Precisión }\end{array}$ & $-11.47^{* *}$ & -6.38 & 0.56 \\
\hline & $\begin{array}{l}\text { Lectura de oraciones. } \\
\text { Comprensión }\end{array}$ & $-12.00^{* *}$ & -5.02 & 0.42 \\
\hline \multirow[t]{5}{*}{$1^{\circ}$} & $\begin{array}{l}\text { Lectura en voz alta. } \\
\text { Tiempo }\end{array}$ & 1.18 & 49.35 & 41.77 \\
\hline & $\begin{array}{l}\text { Lectura en voz alta. } \\
\text { Errores }\end{array}$ & -.37 & -.80 & 2.16 \\
\hline & $\begin{array}{l}\text { Lectura en voz alta. } \\
\text { Comprensión }\end{array}$ & -.93 & -.97 & 1.04 \\
\hline & $\begin{array}{l}\text { Lectura silenciosa. } \\
\text { Tiempo }\end{array}$ & $2.24^{*}$ & 164.37 & 73.52 \\
\hline & $\begin{array}{l}\text { Lectura silenciosa. } \\
\text { Comprensión }\end{array}$ & 1.40 & .94 & 0.67 \\
\hline
\end{tabular}




\begin{tabular}{lccc}
\hline \multicolumn{1}{c}{ Pruebas de lectura } & $t$ & $\begin{array}{c}\text { Diferencia } \\
\text { de medias }\end{array}$ & $\begin{array}{c}\text { Error } \\
\text { típico de la } \\
\text { diferencia }\end{array}$ \\
\hline Lectura de sílabas & $-2.54^{* *}$ & -.38 & 0.15 \\
Lectura de palabras & $-2.03^{*}$ & -.24 & 0.12 \\
$\begin{array}{l}\text { Lectura de no palabras } \\
\text { Lectura de oraciones. }\end{array}$ & -.57 & -.10 & 0.17 \\
$\begin{array}{l}\text { Precisión } \\
\text { Lectura de oraciones. }\end{array}$ & $-5.60^{* *}$ & -1.76 & 0.31 \\
Comprensión & & & 0.15 \\
$\begin{array}{l}\text { Lectura en voz alta. } \\
\text { Tiempo }\end{array}$ & $7.36^{* *}$ & 53.84 & 7.31 \\
$\begin{array}{l}\text { Lectura en voz alta. } \\
\text { Errores }\end{array}$ & $5.07^{* *}$ & 3.42 & 0.67 \\
$\begin{array}{l}\text { Lectura en voz alta. } \\
\text { Comprensión }\end{array}$ & $-6.64^{* *}$ & -2.02 & 0.30 \\
$\begin{array}{l}\text { Lectura silenciosa. } \\
\text { Tiempo }\end{array}$ & $5.55^{* *}$ & 47.53 & 8.56 \\
$\begin{array}{l}\text { Lectura silenciosa. } \\
\text { Comprensión }\end{array}$ & -1.73 & -.67 & 0.38 \\
\hline
\end{tabular}

$* p<.05 ; * * p<.01$.

de lectura de oraciones. Además, los alumnos de la escuela privada presentaron tiempos de lectura silenciosa significativamente menores que los de la escuela pública. Sin embargo, en primer año las diferencias no resultan significativas en el rendimiento en las pruebas de lectura de textos, pero debe considerarse que, como se comentó, tan solo completaron esas pruebas entre siete y nueve niños de la escuela pública (entre un 10\% y un 15\%, aproximadamente).

Respecto de las diferencias entre los alumnos de tercer año, se observa que los de las escuelas privadas presentaron un rendimiento significativamente superior en las pruebas de lectura de sílabas y palabras, y en las de comprensión lectora de oraciones y de lectura en voz alta. Asimismo, se identifican diferencias significativas en el tiempo y en los errores de lectura en voz alta, y en el tiempo de lectura silenciosa. Sin embargo, las diferencias no resultan significativas en la lectura de no pa- labras, la precisión en la lectura de oraciones y la comprensión en la lectura silenciosa de un texto.

La tabla 6 muestra los resultados relativos a la magnitud del efecto de las diferencias entre los alumnos de los sistemas público y privado de primer y tercer año escolar. Se consideran pequeños los efectos de .20 , medios los de .50 y grandes los de .80 (Cohen, 1969). El tamaño del efecto fue mayor para los alumnos de primer año que para los de tercero en las tareas de lectura de sílabas, palabras y no palabras, y en las tareas de precisión y comprensión de lectura de oraciones. En cambio, la magnitud del efecto fue superior para los alumnos de tercero que para los de primero en las tareas de lectura en voz alta (en tiempo, errores y comprensión) y de lectura silenciosa (en tiempo).

Tabla 6

Magnitud del efecto de las diferencias entre rendimientos de alumnos de escuelas públicas y privadas de primer y tercer año

\begin{tabular}{lcc}
\hline \multirow{2}{*}{ Pruebas de lectura } & \multicolumn{2}{c}{ Magnitud del efecto } \\
\cline { 2 - 3 } & Primer año & Tercer año \\
\hline Lectura de sílabas & 3.00 & .00 \\
Lectura de palabras & 2.75 & 2.00 \\
Lectura de no palabras & 3.00 & .00 \\
Lectura de oraciones. Precisión & 2.00 & .00 \\
Lectura de oraciones. Comprensión & 3.16 & 2.00 \\
Lectura en voz alta. Tiempo & .43 & 1.49 \\
Lectura en voz alta. Errores & .22 & 1.02 \\
Lectura en voz alta. Comprensión & .50 & 2.00 \\
Lectura silenciosa. Tiempo & .55 & 1.13 \\
Lectura silenciosa. Comprensión & 1.00 & 1.00 \\
\hline
\end{tabular}

\section{Discusión}

En primer lugar, con respecto a los aspectos de la lectura que han sido evaluados, cabe señalar que los procesos involucrados en las tareas de lectura de sílabas, lectura de palabras, no palabras, oracio- 
nes y un texto leído en voz alta y en silencio son procesos léxicos o microprocesos, dado que están directamente relacionados con la decodificación más o menos mecánica del texto. Al respecto, Sternberg (1996) sostiene que los procesos léxicos intervienen en la identificación de letras y palabras y en la activación en la memoria de información relevante referida a esas palabras.

Acerca de las tareas de comprensión de oraciones, de un texto leído en voz alta y de un texto leído en silencio, los procesos que intervienen son procesos semánticos o macroprocesos, considerados de más alto nivel que los procesos léxicos; los procesos involucrados en la comprensión se encargan de extraer los significados, integrar proposiciones en esquemas y realizar inferencias. Respecto de estos procesos, Gunning (1998) y Sternberg (1996) señalan que se usan para entender el texto como una totalidad y requieren un rol activo por parte del lector, quien debe servirse de su acervo cultural y de las metas que se plantee en relación con la lectura del texto.

Debido a la existencia, ya mencionada, de diferencias marcadas en el nivel socioeconómico de los alumnos que asisten a escuelas privadas y de los que concurren al sistema público de enseñanza en Argentina (DiNIECE, 2009), se consideró el tipo de escuela como un indicador del NSEC de los participantes. Al respecto, Rosselli et al. (2006) señalan que los variados factores ambientales complejos asociados con asistir a una escuela pública o a una escuela privada, como el nivel de escolaridad de los padres, influyen en el desempeño en pruebas de lectura. Para profundizar en la estimación del NSEC de los estudiantes se recabó información sobre la clase social, la condición sociocupacional del jefe/a de familia, el nivel educativo de los padres y la situación ocupacional actual del jefe/a de familia de los participantes (véase Torrado, 1992), y se encontraron claras diferencias entre los usuarios del sistema público y los del sistema privado en todas las dimensiones analizadas. Estas diferencias se sintetizan en un mayor porcentaje de familias de clase social baja, junto con un menor nivel educativo alcanzado por los padres o encargados de los alumnos, en la escuela de gestión pública.

Con respecto al desempeño en lectura, se hallaron diferencias en todos los procesos involucrados en ella, especialmente en los alumnos de primer año. Esto se vio claramente evidenciado en el hecho de que solamente entre siete y nueve alumnos de los 61 que conforman la muestra de primer año de la escuela municipal lograron leer y comprender los textos. Al respecto, Rosselli et al. (2006) plantean que la escolarización tiende a reducir las diferencias halladas en los primeros años de escolaridad. En nuestro trabajo, esto puede verse en la ausencia de diferencias significativas entre los alumnos de tercer año de ambos tipos de escuela en la lectura de no palabras, la precisión en la lectura de oraciones - consideradas microprocesos o procesos de bajo nivel — y la comprensión de un texto leído en silencio - considerada un proceso de más alto nivel-. En este sentido, debemos tener en cuenta que los niños de ese grupo se encuentran escolarizados desde hace ya tres años, y que el ejercicio hace disminuir las diferencias en los procesos de acceso léxico, lo cual repercute además en una mejor comprensión de lo leído.

Estos resultados están respaldados por los tamaños del efecto de las diferencias entre los rendimientos de los alumnos. Se verifica que en la mayoría de las tareas que evalúan los microprocesos de la lectura hay una reducción considerable del tamaño del efecto de las diferencias en los alumnos de tercer grado en relación con los de primero. Por ejemplo, la magnitud de la diferencia entre los alumnos de primer año de los dos tipos de escuela en la tarea de lectura de sílabas es muy alta, mientras que esa diferencia en los alumnos de tercer año es nula. Se entiende, por lo tanto, que la reducción de las diferencias se debe a la mejoría del rendimiento de los alumnos de la escuela pública durante esos años de escolaridad. Sin embargo, se debe dejar constancia de que en este estudio no se evaluó la velocidad de la lectura de palabras y de no palabras, y que ello 
constituye una limitación, en la medida en que en lenguas transparentes como el español tiene mayor determinación la velocidad que la precisión de la lectura (Jiménez \& Hernández-Valle, 2000; Serrano \& Defior, 2008), y, en función de ello, tal vez no ha sido posible determinar la existencia de diferencias en el rendimiento de los alumnos de tercer año en lectura de no palabras.

Como posible consecuencia del incremento de las habilidades de decodificación en los niños de la escuela pública, hallamos la reducción en la magnitud de la diferencia en comprensión lectora entre los niños de primer y tercer año. Es decir, cuando los niños de la escuela pública logran dominar los conocimientos básicos implicados en la adquisición de la lectoescritura, no necesitan emplear tantos procesos y recursos cognitivos en la decodificación y pueden reservarlos para el nivel superior de la comprensión. Así, cuando llegan a tercer año, poseen un mayor control sobre los microprocesos o procesos léxicos (al punto de que se eliminan las diferencias con los alumnos de las escuelas privadas), que les permiten obtener un mejor rendimiento en la tarea de comprensión lectora de oraciones. Del mismo modo, su rendimiento se iguala al de los alumnos de las escuelas privadas en la comprensión de lectura silenciosa.

En cambio, en las tareas de lectura en voz alta y en el tiempo de lectura silenciosa hay un aumento considerable de las diferencias entre los alumnos de ambos sistemas. Es probable que, por un lado, estos resultados reflejen un efecto más potente de la escolarización en los alumnos de las escuelas privadas; es decir, por más de que los alumnos de la escuela pública mejoren, los de las escuelas privadas también lo hacen, sobre todo en la lectura en voz alta, y en mayor grado que sus pares de la escuela pública. Por otra parte, la velocidad de lectura, tanto silenciosa como en voz alta, es el predictor más robusto de la comprensión lectora (Roselli et al., 2006), dado que la disposición de un vocabulario amplio y de estrategias de comprensión eficientes produce una disminución sig- nificativa del tiempo de lectura. Como habíamos anticipado, para leer adecuadamente no basta con ser capaz de decodificar un texto o, dicho de otro modo, de automatizar los microprocesos, sino que es imprescindible disponer de estrategias adecuadas para descifrar el significado del texto. En este trabajo se provee evidencia de que la capacidad de comprensión de los estudiantes de escuelas de NSEC más alto es superior a la de los estudiantes de la escuela de menor NSEC.

De este modo, globalmente, los resultados de este trabajo muestran que el desempeño en pruebas de lectura varía en función del tipo de escuela. Así, en prácticamente todas las tareas administradas se encontró un desempeño más bajo en los alumnos de la escuela pública. Nuestros resultados coinciden con los de otras investigaciones que muestran que los niños provenientes de ambientes socioculturales pobres tienen un peor desempeño lector que los niños de entornos más ricos (DiNIECE, 2012; Magnuson, 2007; Molfese et al., 2003; National Assessment of Educational Progress, 1991; Urquijo, 2009; Wigfield \& Asher, 2002). Al respecto, Bradley y Corwyn (2002), Fernández y Salvador (1994) y Ruiz de Miguel (1999) sostienen que el nivel cultural, laboral y de ingresos de la familia repercute en la disponibilidad de recursos materiales vinculados con la lectura.

En suma, los resultados del presente trabajo presentan evidencias empíricas de la existencia de diferencias marcadas en el desempeño en lectura de los alumnos de escuelas públicas y los alumnos de escuelas privadas, en todos los procesos y en casi todas las pruebas administradas. Se debe destacar que en algunas de las pruebas esa diferencia se reduce con el correr de la escolaridad, pero en otras se hace aún más acentuada. Así, la información proveniente del Ministerio de Educación de la República Argentina (DiNIECE, 2009) acerca de que los niños que asisten a escuelas de gestión privada presentan desempeños superiores en tareas de lectura que los niños que asisten a escuelas de gestión pública, se ve confirmada en este trabajo. 
No obstante, cabe señalar que la ausencia de diferencias entre los alumnos de ambos sistemas educativos en algunas pruebas de lectura de bajo y de alto nivel daría cuenta del cumplimiento parcial de la función niveladora de la escuela, en tanto los niños que recién ingresan a ella (primer año escolar) presentan desempeños aún más desiguales. Los estudiantes provenientes de las clases sociales más desfavorecidas ingresan a la escuela con una clara desventaja en el dominio de las habilidades metalingüísticas necesarias para la decodificación lectora. Al cabo de dos años de escolarización, las diferencias de desempeño en pruebas que evalúan procesos de decodificación se diluyen gracias al efecto de la intervención educativa; sin embargo, las diferencias en la capacidad de comprensión lectora se agudizan, con una clara ventaja para los niños de sectores socioeconómicos y culturales más favorecidos, probablemente por la estimulación recibida en el hogar y el inicio temprano en actividades que involucran el uso de habilidades metalingüísticas.

Consecuentemente, a partir de estos resultados, es posible concluir que resulta importante insistir en la escolarización temprana de los niños de sectores socioeconómicos y culturales más desfavorecidos, con el objeto de compensar los déficits que produce la escasa estimulación para la lectura y la pobreza de sus contextos alfabetizadores.

\section{Referencias}

Andrés, M., Canet-Juric, L., Richard's, M., Introzzi, I. \& Urquijo, S. (2010). Disponibilidad de recursos materiales en el hogar y adquisición de habilidades pre-lectoras. Revista Psicología Escolar e Educacional-ABRAPEE, 14(1), 139-148.

Andrés, M., Navarro Guzmán, J., Urquijo, S. \& Cedeño García, M. (2010). Contexto alfabetizador familiar: relaciones con la adquisición de habilidades prelectoras y desempeño lector. European Journal of Education and Psychology, 3(1), 129-140.
Baquero, R. (1996). Vigotsky y el aprendizaje escolar. Buenos Aires: Aique.

Bracken, S. \& Fischel, J. (2008). Family reading behavior and early literacy skills in preschool children from low-income backgrounds. Early Education and Development, 19(1), 45-47.

Bradley, R. \& Corwyn, R. (2002). Socioeconomic status and child development. Annual Review of Psychology, 53, 371-399.

Cohen, J. (1969). Statistical power analysis for the behavioral sciences. New York: Academic Press.

De los Reyes Aragón, C., Lewis Harb, S. \& Peña Ortiz, M. (2008). Estudio de prevalencia de dificultades de lectura en niños escolarizados de 7 años de Barranquilla (Colombia). Psicología desde el Caribe. Universidad del Norte, 22, 37-49.

Defior Citoler, S. (1994). La conciencia fonológica y la adquisición de la lectoescritura. Infancia y Aprendizaje, 67-68, 91-113.

Defior Citoler, S. (1996). Las dificultades de aprendizaje: un enfoque cognitivo. Málaga: Aljibe.

DiNIECE (2009). Operativo Nacional de Evaluación 2007. Informe de resultados. Recuperado de $\mathrm{http}: / /$ diniece.me.gov.ar/images/stories/diniece/ evaluacion_educativa/nacionales/resultados/Estudio $\% 20$ Nacional $\% 20 \mathrm{de} \% 20$ Evaluacion $\% 20$ $\mathrm{y} \% 20$ Consideraciones $\% 20$ conceptuales $\% 20$ ONE\%202007.pdf

DiNIECE (2012). Operativo Nacional de Evaluación $20103^{\circ}$ y $6^{\circ}$ año de la Educación Primaria. Informe de Resultados. Recuperado de http://iniece.me.gov.ar/images/stories/diniece/ evaluacion_educativa/nacionales/resultados/ INFORME\%20DE\%20RESULTADOS $\% 20$ PRIMARIA\%20ONE\%202010.pdf

Farah, M., Shera, D., Savage, J., Betancourt, L., Giannetta, J., Brodsky, N., ... Hurt, H. (2006). Childhood poverty: Specific associations with neurocognitive development. Brain Research, 1110, 166-174.

Fernández, S. \& Salvador, F. (1994). La familia ante el fracaso escolar. Educadores, 36(169), 7-22. 
Fueyo, A. (1990). El fracaso escolar: entre la ideología y la impotencia. Educadores, 153, 25-40.

García Madruga, J. (2006). Lectura y conocimiento. Barcelona: Paidós.

Garton, A. \& Pratt, C. (1991). Aprendizaje y proceso de alfabetización: el desarrollo del lenguaje hablado y escrito. Barcelona: Paidós.

Gee, J. (1999). Critical issues: Reading and de new literacy studies. Reframing the National Academy of Science Report on Reading. Journal of Literacy Research, 31, 355-374.

Gordon, J. \& Greenidge, J. (1999). Europa: El fracaso escolar y sus consecuencias. Organización y Gestión Educativa, 3, 4-8.

Graves, M., Juel, C. \& Graves, B. (2000). Teaching reading in the 21st century ( $3 \mathrm{a}$ ed.). Needham Heights, MA: Allyn \& Bacon.

Guardia, P. (2003). Relaciones entre habilidades de alfabetización emergente y la lectura desde el nivel de transición mayor a primero básico. Psykhé, 12, 63-79.

Gunning, T. (1998). Assessing and Correcting Reading and Writing Difficulties. Boston: Allyn \& Bacon.

Hackman, D. \& Farah, M. (2009). Socioeconomic status and brain development. Trends in Cognitive Sciences, 13, 65-73.

Hallahan, D., Kauffman, J. \& Lloyd, J. (1999). Introduction to learning disabilities (2a ed.). Boston: Allyn \& Bacon.

Hourigan, M. (1994). Literacy as social exchange: Intersections of class, gender, and culture. New York: State University of New York Press.

Jiménez, J. \& Hernández-Valle, I. (2000). Word identification and reading disorders in the Spanish language. Journal of Learning disabilities, 33(1), 44-60.

Lee, C. (1995). A Culturally based cognitive apprenticeship: Teaching African American high School students skills in literary interpretation. Reading Research Quarterly, 30, 608-630.

León, J. \& García Madruga, J. (1991). Memoria y comprensión de textos. En J. Ruiz-Vargas
(Comp.), Psicología de la memoria. Madrid: Alianza.

Lipina, S., Martelli, M., Vuelta, B., Injoque-Ricle, I. \& Colombo, J. (2004). Poverty and executive performance in preschool pupils from Buenos Aires city (República Argentina), Interdisciplinaria, 21(2), 153-193.

Magnuson, K. (2007). Maternal education and children's academic achievement during middle childhood. Developmental Psychology, 43(6), 1497-1512.

Manly, J., Schupf, N., Tang, M., Weiss, C. \& Stern, Y. (2007). Literacy and cognitive decline among ethnically diverse elders. En Y. Stern (Ed.), Cognitive reserve: Theory and applications ( $\mathrm{pp}$. 219-235). New York, London: Taylor \& Francis. Marder, S., Querejeta, M., Piacente, T., Resches, M. \& Urrutia, M. (2005).Características del contexto alfabetizador en familias de diferente nivel socioeconómico: un estudio comparativo. En E. Díez-Villoria, B. Zubiauz \& M. Mayor (Eds.), Estudio sobre la adquisición del lenguaje (pp. 803-818). Salamanca: Universidad de Salamanca.

Matute, E., Rosselli, M., Ardila, A. \& Ostrosky-Solís, F. (2007). Evaluación Neuropsicológica Infantil (ENI): manual de aplicación. México: El Manual Moderno.

Matute, E., Sanz, A., Gumá, E., Rosselli, M. \& Ardila, A. (2009). Influencia del nivel educativo de los padres, el tipo de escuela y el sexo en el desarrollo de la atención y la memoria. Revista Latinoamericana de Psicología, 41(2), 257276.

Moje, E., Dillon, D. \& O’Brien, D. (2000). Re-examining roles of learner, text, and context in secondary literacy. Journal of Educational Research, 93, 165-180.

Molfese, V., Modglin, A. \& Molfese, D. (2003). The role of environment in the development of reading skills: A longitudinal study of preschool and school-age measures. Journal of Learning Disabilities, 36(1), 59-67. 
Moraso, M. \& Duro, E. (2004). Nutrición, desarrollo y alfabetización. Buenos Aires: UNICEF. Recuperado de http://www.unicef.org/argentina/ spanish/ar_insumos_NAD2.pdf

National Assessment of Educational Progress. (1991). The 1989-1990 National Assessment of Reading and Literature. Denver, CO: Author.

Olinghouse, N. \& Graham, S. (2009). The relationship between the discourse knowledge and the writing performance of elementary-grade students. Journal of Educational Psychology, 101, 37-50.

Piacente, T., Granato, L., Talou, C. \& Marder, S. (2000). Desarrollo psicológico y grupo social de pertenencia: el sesgo cultural de los instrumentos de evaluación. Revista Evaluar, 1, 15-28.

Purcell-Gates, V. (1996). Stories, coupons, and the TV Guide: Relationship between home literacy experiences and emergent literacy knowledge. Reading Research Quarterly, 31, 406-428.

Recart-Herrera, M., Mathiesen-De Gregori, M. \& Herrera-Garbarini, M. (2005). Familia del preescolar y su desempeño escolar posterior. Revista Enfoques Educacionales, 7(1), 105-123.

Rosselli, M., Matute, E. \& Ardila, A. (2006). Predictores neuropsicológicos de la lectura en español. Revista de Neurología, 42, 202-210.

Ruiz de Miguel, C. (1999). La familia y su implicación en el desarrollo infantil. Revista Complutense de Educación, 10(1), 289-304.

Scribner, S. \& Cole, M. (1981). The psychology of literacy. Cambridge, M.A.: Harvard University Press.
Serrano, F. \& Defior, S. (2008). Dyslexia speed problems in a transparent orthography. Annals of Dyslexia, 58, 81-95.

Snow, C. (1983). Literacy and language: relationships during the preschool years. Harvard Educational Review, 53(2), 165-189.

Snow, C. \& Beals, D. (2006). Mealtime talk that supports literacy development. New Directions for Child and Adolescents Development, 111, 51-66.

Sternberg, R. (1996). Cognitive psychology. New York: Holt, Rinehart \& Winston.

Street, B. (1984). Literacy in theory and practice. London: Cambridge University Press.

Torrado, S. (1992). Estructura social de la Argentina. Buenos Aires: Ediciones de la Flor.

Urquijo, S. (2009). Aprendizaje de la lectura: diferencias entre escuelas de gestión pública y de gestión privada. Revista Evaluar, 9, 19-34.

Vellutino, F. \& Scanlon, D. (2002). Emergency literacy skills, early instruction and individual differences as determinants or difficulties in learning to read: The case for early intervention. En S. Neuman y D. Dickinson (Eds.), Handbook of early literacy research (pp. 295-321). Londres: The Guildford Press.

Vigotsky, L. (1988). El desarrollo de los procesos psicológicos superiores. México: Crítica Grijalbo.

Wigfield, A. \& Asher, S. (2002). Social and motivational influences on reading. En P. Pearson (Ed.), Handbook of reading research (pp. 423-452). Mahwah, NJ: Lawrence Erlbaum.
Fecha de recepción: 28 de marzo de 2014 fecha de aceptación: 30 de septiembre de 2014 